\title{
Article \\ Blow-Up of Solutions to Fractional-in-Space Burgers-Type Equations
}

\author{
Munirah Alotaibi ${ }^{\dagger}$, Mohamed Jleli ${ }^{\dagger}\left(\mathbb{D}\right.$ and Bessem Samet ${ }^{*}+{ }^{+}$ \\ Department of Mathematics, College of Science, King Saud University, P.O. Box 2455, Riyadh 11451, Saudi Arabia; \\ 438203454@student.ksu.edu.sa (M.A.); jleli@ksu.edu.sa (M.J.) \\ * Correspondence: bsamet@ksu.edu.sa \\ † These authors contributed equally to this work.
}

Abstract: We consider fractional-in-space analogues of Burgers equation and Korteweg-de VriesBurgers equation on bounded domains. Namely, we establish sufficient conditions for finite-time blow-up of solutions to the mentioned equations. The obtained conditions depend on the initial value and the boundary conditions. Some examples are provided to illustrate our obtained results. In the proofs of our main results, we make use of the test function method and some integral inequalities.

Keywords: fractional-in-space Burgers equation; fractional-in-space Korteweg-de Vries-Burgers equation; global solution; blow-up

MSC: 35K55; 35R11; 35B44

check for updates

Citation: Alotaibi, M.; Jleli, M.; Samet, B. Blow-Up of Solutions to Fractional-in-Space Burgers-Type Equations. Fractal Fract. 2021, 5, 249. https://doi.org/10.3390/ fractalfract 5040249

Academic Editors: Giacomo Ascione, Alessandra Meoli and Enrica Pirozzi

Received: 1 November 2021

Accepted: 23 November 2021

Published: 1 December 2021

Publisher's Note: MDPI stays neutral with regard to jurisdictional claims in published maps and institutional affiliations.

Copyright: (C) 2021 by the authors. Licensee MDPI, Basel, Switzerland. This article is an open access article distributed under the terms and conditions of the Creative Commons Attribution (CC BY) license (https:// creativecommons.org/licenses/by/ $4.0 /)$.

\section{Introduction}

The Burgers equation

$$
\partial_{t} u+u \partial_{x} u=v \partial_{x x} u,
$$

where $v>0$ is a certain parameter, is a fundamental partial differential equation arising in many physical problems, such as fluid mechanics, nonlinear acoustics, gas dynamics and traffic flow. Equation (1) was first introduced by Bateman [1]. Later, in [2,3], Burgers used this equation to capture some features of turbulent fluid in a channel caused by the interaction of the opposite effects of convection and diffusion. Since then, Equation (1) is refereed to as the Burgers equation.

The Korteweg-de Vries-Burgers equation,

$$
\partial_{t} u+u \partial_{x} u+\tau \partial_{x x x} u=v \partial_{x x} u,
$$

where $\tau, v>0$ are certain parameters, was introduced by Su and Gardner [4]. This equation arises within the description of various physical phenomena, such as the propagation of waves in shallow water [5], the propagation of waves in an infinitely long thin walled circular cylinder [6], and plasma waves [7].

In [8], Yushkov and Korpusov studied the finite-time blow-up of solutions to (1) and (2) on bounded domains, under certain boundary conditions.

The study of blow-up phenomena for fractional-in-time evolution equations was initiated by Kirane and his collaborators (see e.g., [9-13]). Very recently, Kirane et al. [10] investigated the finite-time blow-up for different kinds of fractional-in-time dispersive equations on bounded domains, including the fractional-in-time Burgers equation and the fractional-in-time Korteweg-de Vries-Burgers equation. For example, for the fractional-intime analogue of (1), namely

$$
\partial_{0 \mid t}^{\alpha} u+u \partial_{x} u=v \partial_{x x} u, \quad(t, x) \in(0, T) \times(0, L),
$$


where $0<\alpha<1$ and $\partial_{0 \mid t}^{\alpha}$ are the time-Caputo fractional derivative of order $\alpha$, Kirane et al. established a maximum principle, when the initial value $u(0, \cdot)$ is sufficiently smooth. Next, they discussed the influence of gradient nonlinearity on the global solvability of (3) under certain boundary conditions.

Problem (3) was also investigated in [14] by Torebek, where he obtained sufficient conditions depending on the initial value $u(0, \cdot)$ and the boundary conditions, for which there does not exist a global solution to (3).

In this paper, motivated by $[10,14]$, we first consider the fractional-in-space analogue of (1) on a bounded interval, namely,

$$
\left\{\begin{array}{l}
\partial_{t} u+\frac{1}{2 \alpha} \partial_{0 \mid x}^{\alpha}\left(u^{2}\right)=v \partial_{0 \mid x}^{\beta} u, \quad(t, x) \in(0, T) \times(0, L), \\
u(0, x)=u_{0}(x), \quad x \in(0, L) .
\end{array}\right.
$$

Here, $L>0,0<T \leq \infty, v>0$ is a constant, $0<\alpha<1,1<\beta<2$ and $\partial_{0 \mid x^{\prime}}^{\sigma} \sigma \in\{\alpha, \beta\}$, is the space-Caputo fractional derivative (with respect to the variable $x$ ) of order $\sigma$. Using the test function method [15] and some integral estimates, we obtain sufficient conditions depending on $u_{0}$ and the boundary conditions, for which a finite-time blow-up occurs for (4). Next, we discuss the finite-time blow-up for the fractional-in-space analogue of (2) on a bounded interval, namely,

$$
\left\{\begin{array}{l}
\partial_{t} u+\frac{1}{2 \alpha} \partial_{0 \mid x}^{\alpha}\left(u^{2}\right)+\tau \partial_{0 \mid x}^{\beta} u=v \partial_{0 \mid x}^{\gamma} u, \quad(t, x) \in(0, T) \times(0, L), \\
u(0, x)=u_{0}(x), \quad x \in(0, L),
\end{array}\right.
$$

where $\tau, v>0$ are constants, $0<\alpha<1,2<\beta<3,1<\gamma<2$, and $\partial_{0 \mid x^{\prime}}^{\sigma} \sigma \in\{\alpha, \beta, \gamma\}$, is the space-Caputo fractional derivative of order $\sigma$.

The rest of the paper is organized as follows. In Section 2, some preliminaries on fractional calculus, and some useful lemmas are provided. In Section 3, we prove a finite-time blow-up result for the fractional-in-space Burgers Equation (4), and provide an example to illustrate our result. Section 4 is devoted to the study of the fractional-in-space Korteweg-de Vries-Burgers Equation (5).

\section{Preliminaries}

Let $L>0$ be fixed. Given $\sigma>0$ and $f \in L^{1}([0, L])$, the left-sided and right-sided Riemann-Liouville fractional integrals of order $\sigma$ of $f$ are defined respectively by:

$$
\left(I_{0}^{\sigma} f\right)(x)=\frac{1}{\Gamma(\sigma)} \int_{0}^{x}(x-y)^{\sigma-1} f(y) d y \quad \text { and } \quad\left(I_{L}^{\sigma} f\right)(x)=\frac{1}{\Gamma(\sigma)} \int_{x}^{L}(y-x)^{\sigma-1} f(y) d y,
$$

for almost everywhere $x \in[0, L]$, where $\Gamma$ denotes the Gamma function.

Given a positive integer $n, n-1<\sigma<n$, and $f \in C^{n}([0, L])$, the (left-sided) Caputo fractional derivative of order $\sigma$ of $f$ is defined by:

$$
\partial_{0 \mid x}^{\sigma} f(x)=\left(I_{0}^{n-\sigma} \frac{d^{n} f}{d x^{n}}\right)(x)=\frac{1}{\Gamma(n-\sigma)} \int_{0}^{x}(x-y)^{n-\sigma-1} \frac{d^{n} f}{d y^{n}}(y) d y,
$$

for all $x \in[0, L]$. We refer the reader to [16] for the definitions above.

The following integration by parts rule will be used later.

Lemma 1 ([16]). Let $\sigma>0, p, q \geq 1$, and $\frac{1}{p}+\frac{1}{q} \leq 1+\sigma(p \neq 1, q \neq 1$, in the case $\left.\frac{1}{p}+\frac{1}{q}=1+\sigma\right)$. If $(f, g) \in L^{p}([0, L]) \times L^{q}([0, L])$, then

$$
\int_{0}^{L}\left(I_{0}^{\sigma} f\right)(x) g(x) d x=\int_{0}^{L} f(x)\left(I_{L}^{\sigma} g\right)(x) d x .
$$


The following lemma can be shown by elementary calculations.

Lemma 2. Let $n$ be a positive integer and

$$
\varphi(x)=(x-L)^{2 n+1}, \quad x \in[0, L] .
$$

For all $\theta \in(0,1)$, there holds:

$$
\begin{aligned}
\left(I_{L}^{\theta} \varphi\right)(x) & =-\frac{(2 n+1) !}{\Gamma(\theta+2 n+2)}(L-x)^{2 n+\theta+1,} \\
\frac{d\left(I_{L}^{\theta} \varphi\right)}{d x}(x) & =\frac{(2 n+1) !}{\Gamma(2 n+\theta+1)}(L-x)^{2 n+\theta}, \\
\frac{d^{2}\left(I_{L}^{\theta} \varphi\right)}{d x^{2}}(x) & =-\frac{(2 n+1) !}{\Gamma(2 n+\theta)}(L-x)^{2 n+\theta-1}, \\
\frac{d^{3}\left(I_{L}^{\theta} \varphi\right)}{d x^{3}}(x) & =\frac{(2 n+1) !}{\Gamma(2 n+\theta-1)}(L-x)^{2 n+\theta-2} .
\end{aligned}
$$

\section{Finite-Time Blow-Up for the Fractional-in-Space Burgers Equation}

In this section, we consider the fractional-in-space Burgers Equation (4). By a solution to (4), we mean a function $u \in C^{1}\left([0, T), C^{2}([0, L])\right)$ satisfying:

$$
\partial_{t} u+\frac{1}{2 \alpha} \partial_{0 \mid x}^{\alpha}\left(u^{2}\right)=v \partial_{0 \mid x}^{\beta} u,
$$

for all $(t, x) \in(0, T) \times(0, L)$, and the initial condition $u(0, \cdot)=u_{0}$. Moreover, if $T=\infty$, then $u$ is said to be a global solution to (4).

Let $\Phi$ be the set of functions $\varphi \in C^{2}([0, L])$ satisfying the following conditions:

$\left(\Phi_{1}\right)\left(I_{L}^{1-\alpha} \varphi\right)_{x} \geq 0$,

$\left(\Phi_{2}\right) 0<\int_{0}^{L} \frac{\left[\left(I_{L}^{2-\beta} \varphi\right)_{x x}\right]^{2}}{\left(I_{L}^{1-\alpha} \varphi\right)_{x}} d x<\infty$,

$\left(\Phi_{3}\right) 0<\int_{0}^{L} \frac{\varphi^{2}}{\left(I_{L}^{1-\alpha} \varphi\right)_{x}} d x<\infty$,

where $(\cdot)_{x}=\frac{d}{d x}$ and $(.)_{x x}=\frac{d^{2}}{d x^{2}}$.

Suppose now that $u \in C^{1}\left([0, T), C^{2}([0, L])\right)$ is a solution to (4). Multiplying the first equation in (4) by $\varphi \in \Phi$ and integrating over $(0, L)$, we obtain:

$$
\frac{d}{d t} \int_{0}^{L} u(t, x) \varphi(x) d x+\frac{1}{2 \alpha} \int_{0}^{L} \partial_{0 \mid x}^{\alpha}\left(u^{2}\right)(t, x) \varphi(x) d x=v \int_{0}^{L} \partial_{0 \mid x}^{\beta} u(t, x) \varphi(x) d x .
$$

On the other hand, by (6), and using Lemma 1, we obtain:

$$
\begin{aligned}
\int_{0}^{L} \partial_{0 \mid x}^{\alpha}\left(u^{2}\right)(t, x) \varphi(x) d x & =\int_{0}^{L}\left(I_{0}^{1-\alpha}\left(u^{2}\right)_{x}\right)(t, x) \varphi(x) d x \\
& =\int_{0}^{L}\left(u^{2}\right)_{x}(t, x)\left(I_{L}^{1-\alpha} \varphi\right)(x) d x
\end{aligned}
$$

Integrating by parts, there holds:

$$
\int_{0}^{L} \partial_{0 \mid x}^{\alpha}\left(u^{2}\right)(t, x) \varphi(x) d x=\left[u^{2}(t, x)\left(I_{L}^{1-\alpha} \varphi\right)(x)\right]_{x=0}^{L}-\int_{0}^{L} u^{2}(t, x)\left(I_{L}^{1-\alpha} \varphi\right)_{x}(x) d x .
$$


Similarly, we have:

$$
\begin{aligned}
\int_{0}^{L} \partial_{0 \mid x}^{\beta} u(t, x) \varphi(x) d x & =\int_{0}^{L}\left(I_{0}^{2-\beta} u_{x x}\right)(t, x) \varphi(x) d x \\
& =\int_{0}^{L} u_{x x}(t, x)\left(I_{L}^{2-\beta} \varphi\right)(x) d x .
\end{aligned}
$$

Integrating by parts, we obtain:

$$
\begin{aligned}
\int_{0}^{L} \partial_{0 \mid x}^{\beta} u(t, x) \varphi(x) d x= & {\left[u_{x}(t, x)\left(I_{L}^{2-\beta} \varphi\right)(x)-u(t, x)\left(I_{L}^{2-\beta} \varphi\right)_{x}(x)\right]_{x=0}^{L} } \\
& +\int_{0}^{L} u(t, x)\left(I_{L}^{2-\beta} \varphi\right)_{x x}(x) d x
\end{aligned}
$$

Thus, combining (7)-(9), there holds:

$$
\begin{aligned}
& \frac{d}{d t} \int_{0}^{L} u(t, x) \varphi(x) d x \\
& =\frac{1}{2 \alpha} \int_{0}^{L} u^{2}(t, x)\left(I_{L}^{1-\alpha} \varphi\right)_{x}(x) d x+v \int_{0}^{L} u(t, x)\left(I_{L}^{2-\beta} \varphi\right)_{x x}(x) d x+\mathcal{B}(u, \varphi)(t)
\end{aligned}
$$

where

$$
\begin{aligned}
& \mathcal{B}(u, \varphi)(t) \\
& =\left[v\left(u_{x}(t, x)\left(I_{L}^{2-\beta} \varphi\right)(x)-u(t, x)\left(I_{L}^{2-\beta} \varphi\right)_{x}(x)\right)-\frac{1}{2 \alpha} u^{2}(t, x)\left(I_{L}^{1-\alpha} \varphi\right)(x)\right]_{x=0}^{L} .
\end{aligned}
$$

On the other hand, thanks to $\left(\Phi_{2}\right)$, we have:

$$
\begin{aligned}
& \int_{0}^{L}\left(u^{2}(t, x)\left(I_{L}^{1-\alpha} \varphi\right)_{x}(x)+2 \alpha v u(t, x)\left(I_{L}^{2-\beta} \varphi\right)_{x x}(x)\right) d x \\
& =\int_{0}^{L}\left(I_{L}^{1-\alpha} \varphi\right)_{x}(x)\left(u(t, x)+\alpha v \frac{\left(I_{L}^{2-\beta} \varphi\right)_{x x}}{\left(I_{L}^{1-\alpha} \varphi\right)_{x}}\right)^{2} d x-\alpha^{2} v^{2} \int_{0}^{L} \frac{\left[\left(I_{L}^{2-\beta} \varphi\right)_{x x}\right]^{2}}{\left(I_{L}^{1-\alpha} \varphi\right)_{x}} d x
\end{aligned}
$$

that is,

$$
\begin{aligned}
& \int_{0}^{L}\left(u^{2}(t, x)\left(I_{L}^{1-\alpha} \varphi\right)_{x}(x)+2 \alpha v u(t, x)\left(I_{L}^{2-\beta} \varphi\right)_{x x}(x)\right) d x \\
& =\int_{0}^{L} \xi^{2}(t, x)\left(I_{L}^{1-\alpha} \varphi\right)_{x}(x) d x-\alpha^{2} v^{2} \int_{0}^{L} \frac{\left[\left(I_{L}^{2-\beta} \varphi\right)_{x x}\right]^{2}}{\left(I_{L}^{1-\alpha} \varphi\right)_{x}} d x
\end{aligned}
$$

where

$$
\xi(t, x)=u(t, x)+\alpha v \frac{\left(I_{L}^{2-\beta} \varphi\right)_{x x}}{\left(I_{L}^{1-\alpha} \varphi\right)_{x}} .
$$

Using $\left(\Phi_{1}\right),\left(\Phi_{3}\right)$, and Cauchy-Schwarz inequality, we obtain:

$$
\begin{aligned}
\left(\int_{0}^{L} \xi(t, x) \varphi(x) d x\right)^{2} & =\left(\int_{0}^{L} \xi(t, x) \sqrt{\left(I_{L}^{1-\alpha} \varphi\right)_{x}(x)} \frac{\varphi(x)}{\sqrt{\left(I_{L}^{1-\alpha} \varphi\right)_{x}(x)}} d x\right)^{2} \\
& \leq\left(\int_{0}^{L} \xi^{2}(t, x)\left(I_{L}^{1-\alpha} \varphi\right)_{x}(x) d x\right)\left(\int_{0}^{L} \frac{\varphi^{2}(x)}{\left(I_{L}^{1-\alpha} \varphi\right)_{x}(x)} d x\right)
\end{aligned}
$$


which yields

$$
\int_{0}^{L} \xi^{2}(t, x)\left(I_{L}^{1-\alpha} \varphi\right)_{x}(x) d x \geq\left(\int_{0}^{L} \xi(t, x) \varphi(x) d x\right)^{2}\left(\int_{0}^{L} \frac{\varphi^{2}(x)}{\left(I_{L}^{1-\alpha} \varphi\right)_{x}(x)} d x\right)^{-1} .
$$

Thus, it follows from (10), (12), and (14) that:

$$
\begin{aligned}
\frac{d}{d t} \int_{0}^{L} u(t, x) \varphi(x) d x \geq & \frac{1}{2 \alpha}\left(\int_{0}^{L} \frac{\varphi^{2}(x)}{\left(I_{L}^{1-\alpha} \varphi\right)_{x}(x)} d x\right)^{-1}\left(\int_{0}^{L} \xi(t, x) \varphi(x) d x\right)^{2} \\
& -\frac{\alpha v^{2}}{2} \int_{0}^{L} \frac{\left[\left(I_{L}^{2-\beta} \varphi\right)_{x x}\right]^{2}}{\left(I_{L}^{1-\alpha} \varphi\right)_{x}} d x+\mathcal{B}(u, \varphi)(t) .
\end{aligned}
$$

Observe that by (13), we have:

$$
\frac{d}{d t} \int_{0}^{L} u(t, x) \varphi(x) d x=\frac{d}{d t} \int_{0}^{L} \xi(t, x) \varphi(x) d x .
$$

Hence, there holds:

$$
\begin{aligned}
\frac{d}{d t} \int_{0}^{L} \xi(t, x) \varphi(x) d x \geq & \frac{1}{2 \alpha}\left(\int_{0}^{L} \frac{\varphi^{2}(x)}{\left(I_{L}^{1-\alpha} \varphi\right)_{x}(x)} d x\right)^{-1}\left(\int_{0}^{L} \xi(t, x) \varphi(x) d x\right)^{2} \\
& -\frac{\alpha v^{2}}{2} \int_{0}^{L} \frac{\left[\left(I_{L}^{2-\beta} \varphi\right)_{x x}\right]^{2}}{\left(I_{L}^{1-\alpha} \varphi\right)_{x}} d x+\mathcal{B}(u, \varphi)(t) .
\end{aligned}
$$

Suppose that $\mathcal{B}(u, \varphi)(t) \geq 0$ for all $t$. Then, the above inequality yields:

$$
\frac{d F}{d t}(t) \geq \rho^{2} F^{2}(t)-\mu^{2}
$$

where

$$
\begin{aligned}
F(t) & =\int_{0}^{L} \xi(t, x) \varphi(x) d x, \\
\rho & =\frac{1}{\sqrt{2 \alpha}}\left(\int_{0}^{L} \frac{\varphi^{2}(x)}{\left(I_{L}^{1-\alpha} \varphi\right)_{x}(x)} d x\right)^{\frac{-1}{2}}, \\
\mu & =\frac{v \sqrt{\alpha}}{\sqrt{2}}\left(\int_{0}^{L} \frac{\left[\left(I_{L}^{2-\beta} \varphi\right)_{x x}\right]^{2}}{\left(I_{L}^{1-\alpha} \varphi\right)_{x}} d x\right)^{\frac{1}{2}} .
\end{aligned}
$$

Hence, from the theory of ordinary differential equations, we deduce the following finite-time blow-up result for (4).

Theorem 1. Let $u \in C^{1}\left([0, T), C^{2}([0, L])\right.$ be a solution to (4), such that $\mathcal{B}(u, \varphi) \geq 0$ for some $\varphi \in \Phi$. Suppose that $u_{0} \in L^{1}([0, L])$ and

$$
F_{0}:=F(0)=\int_{0}^{L}\left(u_{0}+\alpha v \frac{\left(I_{L}^{2-\beta} \varphi\right)_{x x}}{\left(I_{L}^{1-\alpha} \varphi\right)_{x}}\right) \varphi(x) d x>\frac{\mu}{\rho} .
$$

Then the following estimate holds:

$$
F(t) \geq \frac{\mu}{\rho} \frac{1+k_{0} \exp (2 \mu \rho t)}{1-k_{0} \exp (2 \mu \rho t)}, \quad k_{0}=\frac{\rho F_{0}-\mu}{\rho F_{0}+\mu},
$$


and hence $T \leq T^{*}$, where

$$
T^{*}=-\frac{1}{2 \mu \rho} \ln k_{0} .
$$

Moreover, if $T=T^{*}$, then $\lim _{t \rightarrow T^{-}} F(t)=+\infty$.

We provide below an example to illustrate our obtained result.

Example 1. Consider problem (4) with $L=1$, under the boundary conditions

$$
\left.u\right|_{x=0}=\left.u_{x}\right|_{x=0}=0 .
$$

Let

$$
\varphi(x)=(x-1)^{3}, \quad x \in[0,1] .
$$

First, let us check that the function $\varphi$ belongs to $\Phi$. Using Lemma 2 with $n=1, \theta=1-\alpha$, and $L=1$, we obtain:

$$
\left(I_{1}^{1-\alpha} \varphi\right)_{x}(x)=\frac{6}{\Gamma(4-\alpha)}(1-x)^{3-\alpha},
$$

which shows that the function $\varphi$ satisfies condition $\left(\Phi_{1}\right)$. Again, using Lemma 2 with $n=1$, $\theta=2-\beta$, and $L=1$, we obtain:

$$
\left(I_{1}^{2-\beta} \varphi\right)_{x x}(x)=-\frac{6}{\Gamma(4-\beta)}(1-x)^{3-\beta} .
$$

By (17) and (18), there holds:

$$
\frac{\left[\left(I_{1}^{2-\beta} \varphi\right)_{x x}\right]^{2}}{\left(I_{1}^{1-\alpha} \varphi\right)_{x}}=\frac{6 \Gamma(4-\alpha)}{\Gamma^{2}(4-\beta)}(1-x)^{3-2 \beta+\alpha} .
$$

Integrating over $(0,1)$, we obtain:

$$
\int_{0}^{1} \frac{\left[\left(I_{1}^{2-\beta} \varphi\right)_{x x}\right]^{2}}{\left(I_{1}^{1-\alpha} \varphi\right)_{x}} d x=\frac{6 \Gamma(4-\alpha)}{(\alpha-2 \beta+4) \Gamma^{2}(4-\beta)},
$$

which shows that the function $\varphi$ satisfies condition $\left(\Phi_{2}\right)$. Next, by (17), we obtain:

$$
\frac{\varphi^{2}(x)}{\left(I_{1}^{1-\alpha} \varphi\right)_{x}(x)}=\frac{\Gamma(4-\alpha)}{6}(1-x)^{3+\alpha} .
$$

Integrating over $(0,1)$, we get:

$$
\int_{0}^{1} \frac{\varphi^{2}}{\left(I_{1}^{1-\alpha} \varphi\right)_{x}} d x=\frac{\Gamma(4-\alpha)}{6(4+\alpha)}
$$

which shows that condition $\left(\Phi_{3}\right)$ is satisfied by the function $\varphi$. Consequently, we have $\varphi \in \Phi$.

Moreover, by (19) and (20), we obtain:

$$
\rho=\sqrt{\frac{3(4+\alpha)}{\alpha \Gamma(4-\alpha)}}
$$

and

$$
\mu=\frac{v}{\Gamma(4-\beta)} \sqrt{\frac{3 \alpha \Gamma(4-\alpha)}{\alpha-2 \beta+4}} .
$$


By (17) and (18), we get

$$
\begin{aligned}
\int_{0}^{1} \frac{\left(I_{1}^{2-\beta} \varphi\right)_{x x}}{\left(I_{1}^{1-\alpha} \varphi\right)_{x}} \varphi(x) d x & =\frac{\Gamma(4-\alpha)}{\Gamma(4-\beta)} \int_{0}^{1}(1-x)^{\alpha-\beta+3} d x \\
& =\frac{\Gamma(4-\alpha)}{(4+\alpha-\beta) \Gamma(4-\beta)} .
\end{aligned}
$$

Hence, (15) is equivalent to:

$$
\int_{0}^{1} u_{0}(x)(x-1)^{3} d x>\frac{\alpha v \Gamma(4-\alpha)}{\Gamma(4-\beta)}\left[\frac{1}{\sqrt{(4+\alpha)(4+\alpha-2 \beta)}}-\frac{1}{4+\alpha-\beta}\right] .
$$

On the other hand, observe that, if $u$ is a solution to (4)-(16), then by (11),

$$
\begin{aligned}
& \mathcal{B}(u, \varphi)(t) \\
& =\left[v\left(u_{x}(t, x)\left(I_{1}^{2-\beta} \varphi\right)(x)-u(t, x)\left(I_{1}^{2-\beta} \varphi\right)_{x}(x)\right)-\frac{1}{2 \alpha} u^{2}(t, x)\left(I_{1}^{1-\alpha} \varphi\right)(x)\right]_{x=0}^{1} \\
& =0 .
\end{aligned}
$$

Thus, by Theorem 1, we deduce that, if $u_{0} \in L^{1}([0,1])$ and (21) holds, then (4)-(16) admits no global solution.

\section{Finite-Time Blow-Up for the Fractional-in-Space Korteweg-de Vries-Burgers Equation}

In this section, we consider the Korteweg-de Vries-Burgers Equation (5). By a solution to (5), we mean a function $u \in C^{1}\left([0, T), C^{3}([0, L])\right)$ satisfying:

$$
\partial_{t} u+\frac{1}{2 \alpha} \partial_{0 \mid x}^{\alpha}\left(u^{2}\right)+\tau \partial_{0 \mid x}^{\beta} u=v \partial_{0 \mid x}^{\gamma} u,
$$

for all $(t, x) \in(0, T) \times(0, L)$, and the initial condition $u(0, \cdot)=u_{0}$. If $T=\infty$, then $u$ is said to be a global solution to (5).

Let $\Psi$ be the set of functions $\psi \in C^{3}([0, L])$ satisfying the following conditions:

$\left(\Psi_{1}\right)\left(I_{L}^{1-\alpha} \psi\right)_{x} \geq 0$,

$\left(\Psi_{2}\right) 0<\int_{0}^{L} \frac{\psi^{2}}{\left(I_{L}^{1-\alpha} \psi\right)_{x}} d x<\infty$,

$\left(\Psi_{3}\right) 0<\int_{0}^{L} \frac{\left(v\left(I_{L}^{2-\gamma} \psi\right)_{x x}+\tau\left(I_{L}^{3-\beta} \psi\right)_{x x x}\right)^{2}}{\left(I_{L}^{1-\alpha} \psi\right)_{x}} d x<\infty$.

Suppose that $u \in C^{1}\left([0, T), C^{3}([0, L])\right)$ is a solution to (5). Multiplying the first equation in (5) by $\psi \in \Psi$ and integrating over $(0, L)$, we obtain:

$$
\begin{aligned}
& \frac{d}{d t} \int_{0}^{L} u(t, x) \psi(x) d x+\frac{1}{2 \alpha} \int_{0}^{L} \partial_{0 \mid x}^{\alpha}\left(u^{2}\right)(t, x) \psi(x) d x+\tau \int_{0}^{L} \partial_{0 \mid x}^{\beta} u(t, x) \psi(x) d x \\
& =v \int_{0}^{L} \partial_{0 \mid x}^{\gamma} u(t, x) \psi(x) d x .
\end{aligned}
$$

As previously, using Lemma 1, and integrating by parts, we obtain:

$$
\int_{0}^{L} \partial_{0 \mid x}^{\alpha}\left(u^{2}\right)(t, x) \psi(x) d x=\left[u^{2}(t, x)\left(I_{L}^{1-\alpha} \psi\right)(x)\right]_{x=0}^{L}-\int_{0}^{L} u^{2}(t, x)\left(I_{L}^{1-\alpha} \psi\right)_{x}(x) d x,
$$




$$
\begin{aligned}
\int_{0}^{L} \partial_{0 \mid x}^{\gamma} u(t, x) \psi(x) d x= & {\left[u_{x}(t, x)\left(I_{L}^{2-\gamma} \psi\right)(x)-u(t, x)\left(I_{L}^{2-\gamma} \psi\right)_{x}(x)\right]_{x=0}^{L} } \\
& +\int_{0}^{L} u(t, x)\left(I_{L}^{2-\gamma} \psi\right)_{x x}(x) d x
\end{aligned}
$$

and

$$
\begin{aligned}
& \int_{0}^{L} \partial_{0 \mid x}^{\beta} u(t, x) \psi(x) d x \\
& =\left[u_{x x}(t, x)\left(I_{L}^{3-\beta} \psi\right)(x)-u_{x}(t, x)\left(I_{L}^{3-\beta} \psi\right)_{x}(x)+u(t, x)\left(I_{L}^{3-\beta} \psi\right)_{x x}(x)\right]_{x=0}^{L} \\
& -\int_{0}^{L} u(t, x)\left(I_{L}^{3-\beta} \psi\right)_{x x x}(x) d x .
\end{aligned}
$$

Combining (22)-(25), there holds:

$$
\begin{aligned}
\frac{d}{d t} \int_{0}^{L} u(t, x) \psi(x) d x= & \frac{1}{2 \alpha} \int_{0}^{L} u^{2}(t, x)\left(I_{L}^{1-\alpha} \psi\right)_{x}(x) d x+\tau \int_{0}^{L} u(t, x)\left(I_{L}^{3-\beta} \psi\right)_{x x x}(x) d x \\
& +v \int_{0}^{L} u(t, x)\left(I_{L}^{2-\gamma} \psi\right)_{x x}(x) d x+\mathcal{B}(u, \psi)(t)
\end{aligned}
$$

$\mathcal{B}(u, \psi)(t)$

where

$$
\begin{aligned}
& =\left[u_{x}(t, x)\left(v\left(I_{L}^{2-\gamma} \psi\right)(x)+\tau\left(I_{L}^{3-\beta} \psi\right)_{x}(x)\right)-u(t, x)\left(v\left(I_{L}^{2-\gamma} \psi\right)_{x}(x)+\tau\left(I_{L}^{3-\beta} \psi\right)_{x x}(x)\right)\right]_{x=0}^{L} \\
& -\left[\tau u_{x x}(t, x)\left(I_{L}^{3-\beta} \psi\right)(x)+\frac{1}{2 \alpha} u^{2}(t, x)\left(I_{L}^{1-\alpha} \psi\right)(x)\right]_{x=0}^{L} .
\end{aligned}
$$

On the other hand, thanks to $\left(\Psi_{3}\right)$, we have:

$$
\begin{aligned}
& \int_{0}^{L}\left(u^{2}(t, x)\left(I_{L}^{1-\alpha} \psi\right)_{x}(x)+2 \alpha \tau u(t, x)\left(I_{L}^{3-\beta} \psi\right)_{x x x}(x)+2 \alpha v u(t, x)\left(I_{L}^{2-\gamma} \psi\right)_{x x}(x)\right) d x \\
& =\int_{0}^{L}\left(I_{L}^{1-\alpha} \psi\right)_{x}(x)\left(u^{2}(t, x)+2 u(t, x)\left(\alpha \tau \frac{\left(I_{L}^{3-\beta} \psi\right)_{x x x}(x)}{\left(I_{L}^{1-\alpha} \psi\right)_{x}(x)}+\alpha v \frac{\left(I_{L}^{2-\gamma} \psi\right)_{x x}(x)}{\left(I_{L}^{1-\alpha} \psi\right)_{x}(x)}\right)\right) d x \\
& =\int_{0}^{L}\left(I_{L}^{1-\alpha} \psi\right)_{x}(x)\left(u(t, x)+\alpha \tau \frac{\left(I_{L}^{3-\beta} \psi\right)_{x x x}(x)}{\left(I_{L}^{1-\alpha} \psi\right)_{x}(x)}+\alpha v \frac{\left(I_{L}^{2-\gamma} \psi\right)_{x x}(x)}{\left(I_{L}^{1-\alpha} \psi\right)_{x}(x)}\right)^{2} d x \\
& -\alpha^{2} \int_{0}^{L} \frac{\left(v\left(I_{L}^{2-\gamma} \psi\right)_{x x}+\tau\left(I_{L}^{3-\beta} \psi\right)_{x x x}\right)^{2}}{\left(I_{L}^{1-\alpha} \psi\right)_{x}} d x
\end{aligned}
$$

that is,

$$
\begin{aligned}
& \int_{0}^{L}\left(u^{2}(t, x)\left(I_{L}^{1-\alpha} \psi\right)_{x}(x)+2 \alpha \tau u(t, x)\left(I_{L}^{3-\beta} \psi\right)_{x x x}(x)+2 \alpha v u(t, x)\left(I_{L}^{2-\gamma} \psi\right)_{x x}(x)\right) d x \\
& =\int_{0}^{L} \vartheta^{2}(t, x)\left(I_{L}^{1-\alpha} \psi\right)_{x}(x) d x-\alpha^{2} \int_{0}^{L} \frac{\left(v\left(I_{L}^{2-\gamma} \psi\right)_{x x}+\tau\left(I_{L}^{3-\beta} \psi\right)_{x x x}\right)^{2}}{\left(I_{L}^{1-\alpha} \psi\right)_{x}} d x
\end{aligned}
$$

where

$$
\vartheta(t, x)=u(t, x)+\alpha \frac{\tau\left(I_{L}^{3-\beta} \psi\right)_{x x x}(x)+v\left(I_{L}^{2-\gamma} \psi\right)_{x x}(x)}{\left(I_{L}^{1-\alpha} \psi\right)_{x}(x)} .
$$

Next, using $\left(\Psi_{1}\right),\left(\Psi_{2}\right)$, and Cauchy-Schwarz inequality, we obtain:

$$
\left(\int_{0}^{L} \vartheta(t, x) \psi(x) d x\right)^{2} \leq\left(\int_{0}^{L} \vartheta^{2}(t, x)\left(I_{L}^{1-\alpha} \psi\right)_{x}(x) d x\right)\left(\int_{0}^{L} \frac{\psi^{2}(x)}{\left(I_{L}^{1-\alpha} \psi\right)_{x}(x)} d x\right)
$$


which yields

$$
\int_{0}^{L} \vartheta^{2}(t, x)\left(I_{L}^{1-\alpha} \psi\right)_{x}(x) d x \geq\left(\int_{0}^{L} \vartheta(t, x) \psi(x) d x\right)^{2}\left(\int_{0}^{L} \frac{\psi^{2}(x)}{\left(I_{L}^{1-\alpha} \psi\right)_{x}(x)} d x\right)^{-1} .
$$

Thus, by (26), (28)-(30), we obtain:

$$
\begin{aligned}
\frac{d}{d t} \int_{0}^{L} \vartheta(t, x) \psi(x) d x \geq & \frac{1}{2 \alpha}\left(\int_{0}^{L} \frac{\psi^{2}(x)}{\left(I_{L}^{1-\alpha} \psi\right)_{x}(x)} d x\right)^{-1}\left(\int_{0}^{L} \vartheta(t, x) \psi(x) d x\right)^{2} \\
& -\frac{\alpha}{2} \int_{0}^{L} \frac{\left(v\left(I_{L}^{2-\gamma} \psi\right)_{x x}+\tau\left(I_{L}^{3-\beta} \psi\right)_{x x x}\right)^{2}}{\left(I_{L}^{1-\alpha} \psi\right)_{x}} d x+\mathcal{B}(u, \psi)(t) .
\end{aligned}
$$

Suppose that $\mathcal{B}(u, \varphi)(t) \geq 0$ for all $t$. Then, the above inequality yields:

$$
\frac{d F}{d t}(t) \geq \rho^{2} F^{2}(t)-\mu^{2},
$$

where

$$
\begin{aligned}
F(t) & =\int_{0}^{L} \vartheta(t, x) \psi(x) d x, \\
\rho & =\frac{1}{\sqrt{2 \alpha}}\left(\int_{0}^{L} \frac{\psi^{2}(x)}{\left(I_{L}^{1-\alpha} \psi\right)_{x}(x)} d x\right)^{\frac{-1}{2}}, \\
\mu & =\frac{\sqrt{\alpha}}{\sqrt{2}}\left(\int_{0}^{L} \frac{\left(v\left(I_{L}^{2-\gamma} \psi\right)_{x x}+\tau\left(I_{L}^{3-\beta} \psi\right)_{x x x}\right)^{2}}{\left(I_{L}^{1-\alpha} \psi\right)_{x}} d x\right)^{\frac{1}{2}} .
\end{aligned}
$$

Hence, we deduce the following blow-up result for (5).

Theorem 2. Let $u \in C^{1}\left([0, T), C^{3}([0, L])\right.$ be a solution to (5) such that $\mathcal{B}(u, \psi) \geq 0$ for some $\psi \in \Psi$. Suppose that $u_{0} \in L^{1}([0, L])$ and

$$
F_{0}:=F(0)=\int_{0}^{L}\left(u_{0}(x)+\alpha \frac{\tau\left(I_{L}^{3-\beta} \psi\right)_{x x x}(x)+v\left(I_{L}^{2-\gamma} \psi\right)_{x x}(x)}{\left(I_{L}^{1-\alpha} \psi\right)_{x}(x)}\right) \psi(x) d x>\frac{\mu}{\rho} .
$$

Then, the result of Theorem 1 holds.

An example is provided below to illustrate the above result.

Example 2. Consider problem (5) with $L=1$, under the boundary conditions:

$$
\left.u\right|_{x=0}=2 \alpha \Gamma(7-\alpha)\left(\frac{v}{\Gamma(7-\gamma)}-\frac{\tau}{\Gamma(7-\beta)}\right),\left.\quad u_{x}\right|_{x=0}=\left.u_{x x}\right|_{x=0}=0 .
$$

Let

$$
\psi(x)=(x-1)^{5}, \quad x \in[0,1] .
$$

Let us check that the function $\psi$ belongs to $\Psi$. Using Lemma 2 with $n=2, \theta=1-\alpha$, and $L=1$, we obtain:

$$
\left(I_{1}^{1-\alpha} \psi\right)_{x}(x)=\frac{120}{\Gamma(6-\alpha)}(1-x)^{5-\alpha},
$$


which shows that the function $\psi$ satisfies condition $\left(\Psi_{1}\right)$. Using Lemma 2 with $n=2, \theta=2-\gamma$, and $L=1$, we obtain:

$$
\left(I_{1}^{2-\gamma} \psi\right)_{x x}(x)=-\frac{120}{\Gamma(6-\gamma)}(1-x)^{5-\gamma} .
$$

Again, using Lemma 2 with $n=2, \theta=3-\beta$, and $L=1$, we obtain:

$$
\left(I_{1}^{3-\beta} \psi\right)_{x x x}(x)=\frac{120}{\Gamma(6-\beta)}(1-x)^{5-\beta} .
$$

By (33), there holds:

$$
\int_{0}^{1} \frac{\psi^{2}}{\left(I_{1}^{1-\alpha} \psi\right)_{x}} d x=\frac{\Gamma(6-\alpha)}{120(6+\alpha)}
$$

which shows that the function $\psi$ satisfies condition $\left(\Psi_{2}\right)$. Next, using (33)-(35), an elementary calculation shows that:

$$
\begin{aligned}
& \int_{0}^{1} \frac{\left(v\left(I_{1}^{2-\gamma} \psi\right)_{x x}+\tau\left(I_{1}^{3-\beta} \psi\right)_{x x x}\right)^{2}}{\left(I_{1}^{1-\alpha} \psi\right)_{x}} d x \\
& =120 \Gamma(6-\alpha) \\
& \times\left(\frac{v^{2}}{(\alpha-2 \gamma+6) \Gamma^{2}(6-\gamma)}+\frac{\tau^{2}}{(\alpha-2 \beta+6) \Gamma^{2}(6-\beta)}-\frac{2 v \tau}{(\alpha-\gamma-\beta+6) \Gamma(6-\beta) \Gamma(6-\gamma)}\right),
\end{aligned}
$$

which proves that condition $\left(\Psi_{3}\right)$ is satisfied by the function $\psi$. Consequently, we have $\psi \in \Psi$.

The parameters $\rho$ and $\mu$ can be obtained using (36) and (37). Namely, we have:

$$
\rho=\sqrt{\frac{60(6+\alpha)}{\alpha \Gamma(6-\alpha)}}
$$

and

$$
\begin{aligned}
& \frac{\mu}{\sqrt{60 \alpha \Gamma(6-\alpha)}} \\
& =\left(\frac{v^{2}}{(\alpha-2 \gamma+6) \Gamma^{2}(6-\gamma)}+\frac{\tau^{2}}{(\alpha-2 \beta+6) \Gamma^{2}(6-\beta)}-\frac{2 v \tau}{(\alpha-\gamma-\beta+6) \Gamma(6-\beta) \Gamma(6-\gamma)}\right)^{\frac{1}{2}} .
\end{aligned}
$$

Moreover, by (33)-(35), an elementary calculation shows that:

$$
\begin{aligned}
& \int_{0}^{1} \frac{\tau\left(I_{1}^{3-\beta} \psi\right)_{x x x}(x)+v\left(I_{1}^{2-\gamma} \psi\right)_{x x}(x)}{\left(I_{1}^{1-\alpha} \psi\right)_{x}(x)} \psi(x) d x \\
& =\Gamma(6-\alpha)\left(\frac{v}{(6+\alpha-\gamma) \Gamma(6-\gamma)}-\frac{\tau}{(6+\alpha-\beta) \Gamma(6-\beta)}\right) .
\end{aligned}
$$

Then, (31) is equivalent to:

$$
\begin{aligned}
& \frac{1}{\alpha} \int_{0}^{1} u_{0}(x)(x-1)^{5} d x \\
& >\frac{\Gamma(6-\alpha)}{\sqrt{6+\alpha}} \\
& \times\left(\frac{v^{2}}{(\alpha-2 \gamma+6) \Gamma^{2}(6-\gamma)}+\frac{\tau^{2}}{(\alpha-2 \beta+6) \Gamma^{2}(6-\beta)}-\frac{2 v \tau}{(\alpha-\gamma-\beta+6) \Gamma(6-\beta) \Gamma(6-\gamma)}\right)^{\frac{1}{2}} \\
& -\Gamma(6-\alpha)\left(\frac{v}{(6+\alpha-\gamma) \Gamma(6-\gamma)}-\frac{\tau}{(6+\alpha-\beta) \Gamma(6-\beta)}\right) .
\end{aligned}
$$


On the other hand, observe that, if $u$ is a solution to (5)-(32), then by (27) and the previous calculations, we obtain:

$$
\begin{aligned}
\mathcal{B}(u, \psi)(t) & =60 u(t, 0)\left(\frac{2 v}{\Gamma(7-\gamma)}-\frac{2 \tau}{\Gamma(7-\beta)}-\frac{u(t, 0)}{\alpha \Gamma(7-\alpha)}\right) \\
& =0
\end{aligned}
$$

Thus, by Theorem 2, we deduce that, if $u_{0} \in L^{1}([0,1])$ and (38) holds, then (5)-(32) admits no global solution.

\section{Conclusions}

Fractional-in-space Burgers-type equations are investigated in this paper. For the fractional-in-space Burgers Equation (4), we proved that, if $u_{0} \in L^{1}([0, L]), \mathcal{B}(u, \varphi) \geq 0$ for some $\varphi \in \Phi$, and (15) holds, then a finite-time blow-up occurs. For the fractional-in-space Korteweg-de Vries-Burgers Equation (5), we established that, if $u_{0} \in L^{1}([0, L]), \mathcal{B}(u, \psi) \geq 0$ for some $\psi \in \Psi$, and (31) holds, then the same conclusion as above holds.

In this paper, we focused only on the large time behavior of solutions to the considered problems. Namely, it is supposed that problems (4) and (5) admit local solutions. It will be interesting to investigate the local solvability for the mentioned problems.

Author Contributions: Investigation, M.A.; Supervision M.J. and B.S. All authors have read and agreed to the published version of the manuscript.

Funding: The second author is supported by the Researchers Supporting Project number (RSP2021/57), King Saud University, Riyadh, Saudi Arabia.

Institutional Review Board Statement: Not applicable.

Informed Consent Statement: Not applicable.

Data Availability Statement: Not applicable.

Conflicts of Interest: The authors declare no conflict of interest.

\section{References}

1. Bateman, H. Some recent researches on the motion of fluids. Monthly Weather Rev. 1915, 43, 163-170. [CrossRef]

2. Burgers, J.M. Mathematical examples illustrating relations occurring in the theory of turbulent fluid motion. Trans. R. Neth. Acad. Sci. Amst. 1939, 17, 1-53.

3. Burgers, J.M. A mathematical model illustrating the theory of turbulence. Adv. Appl. Mech. 1948, 1, 171-199.

4. Su, C.H.; Gardner, C.S. Derivation of the Korteweg-de-Vries and Burgers' equation. J. Math. Phys. 1969, 10, 536-539. [CrossRef]

5. Jonson, R.S. A nonlinear equation incorporating damping and dispersion. J. Phys. Mech. 1970, 42, 49-60.

6. Murawski, K. The Korteweg-de Vries equation for wave propagation in an infinitely long thin walled circular cylinder obtained via the Lagrangian method. Z. Nat. A 1985, 40, 955-956. [CrossRef]

7. Grad, H.; Hu, P.N. Unified shock profile in a plasma. Phys. Mech. 1967, 10, 2596-2601. [CrossRef]

8. Yushkov, E.V.; Korpusov, M.O. Global unsolvability of one-dimensional problems for Burgers-type equations. Math. Notes 2015, 98, 503-514. [CrossRef]

9. Alsaedi, A.; Ahmad, B.; Kirane, M.; Musalhi, F.; Alzahrani, F. Blowing-up solutions for a nonlinear time-fractional system. Bull. Math. Sci. 2017, 7, 201-210. [CrossRef]

10. Alsaedi, A.; Ahmad, B.; Kirane, M.; Torebek, B.T. Blowing-up solutions of the time-fractional dispersive equations. Adv. Nonlinear Anal. 2021, 10, 952-971. [CrossRef]

11. Dannawi, I.; Kirane, M.; Fino, A.Z. Finite time blow-up for damped wave equations with space-time dependent potential and nonlinear memory. NoDEA Nonlinear Differ. Equ. Appl. 2018, 25, 1-19. [CrossRef]

12. Fino, A.Z.; Kirane, M. Qualitative properties of solutions to a time-space fractional evolution equation. Q. Appl. Math. 2012, 70, 133-157. [CrossRef]

13. Kirane, M.; Malik, S.A. The profile of blowing-up solutions to a nonlinear system of fractional differential equations. Nonlinear Anal. 2010, 73, 3723-3736. [CrossRef]

14. Torebek, B.T. Global unsolvability of the Burgers equation with fractional time derivative. Differ. Equ. 2019, 55, 867-870. [CrossRef] 
15. Mitidieri, E.; Pohozaev, S. A priori estimates and blow-up of solutions to nonlinear partial differential equations and inequalities. Proc. Steklov. Inst. Math. 2001, 234, 1-383.

16. Samko, S.G.; Kilbas, A.A.; Marichev, O.I. Fractional Integrals and Derivatives: Theory and Applications; Gordon and Breach: Yverdon, Switzerland, 1993. 